\title{
AAA+ ClpB chaperone as a potential virulence factor of pathogenic microorganisms: Other aspect of its chaperone function
}

\author{
Joanna Krajewska, Sabina Kędzierska-Mieszkowska*
}

Department of Biochemistry, University of Gdańsk, Gdańsk, Poland

Email: " sabina.kedzierska-mieszkowska@biol.ug.edu.pl

Received 12 November 2013; revised 13 December 2013; accepted 26 December 2013

Copyright (C) 2014 Joanna Krajewska, Sabina Kędzierska-Mieszkowska. This is an open access article distributed under the Creative Commons Attribution License, which permits unrestricted use, distribution, and reproduction in any medium, provided the original work is properly cited. In accordance of the Creative Commons Attribution License all Copyrights (C) 2014 are reserved for SCIRP and the owner of the intellectual property Joanna Krajewska, Sabina Kędzierska-Mieszkowska. All Copyright (C) 2014 are guarded by law and by SCIRP as a guardian.

\section{ABSTRACT}

We describe and discuss the most recent findings on the activity and function of the oligomeric AAA+ chaperone ClpB from the Hsp100 protein family in pathogenic microorganisms. Pathogens are exposed to significant stress during infection of the host cells, frequently resulting in protein aggregation. The fact that ClpB is usually up-regulated in pathogens together with its immune reactivity suggests that $\mathrm{ClpB}$ acting as a protein disaggregase may be important for pathogen invasion and virulence. However, the specific function of $\mathrm{ClpB}$ in pathogenicity is still unclear. Since it is known that ClpB does not exist in mammals, it may serve as a potential target for the development of an effective therapy against several major bacterial diseases that do not respond to conventional antibiotics.

\section{KEYWORDS}

AAA+ ATPase; ClpB; Molecular Chaperone; Virulence; Pathogens

\section{INTRODUCTION}

\subsection{ClpB, an AAA+ ATPase Chaperone}

Bacterial molecular chaperone $\mathrm{ClpB}$ solubilizes and reactivates stress-agregated proteins in collaboration with the DnaK chaperone system by forming a bichaperone network that is crucial for the development of bacterial thermotolerance [1,2]. Yeast proteins Hsp104 (located in the cytosol) and Hsp78 (found in the mitochondrial ma-

*Corresponding author. trix), and plant Hsp101 are among the eukaryotic homologues of the bacterial ClpB. ClpB is a representative of the Hsp100 protein family that belongs to the AAA+ superfamily of ATPases (ATPases Associated with a variety of cellular Activities) [3]. For example, AAA+ ATPases are involved in assembling and disassembling of large protein complexes which are involved in cellular processes such as replication, recombination and DNA repair, intracellular transport and organelle biogenesis. They also play a key function in the posttranslational protein quality control system that takes care of the state of protein folding and the proper functioning of the cells. AAA+ proteins may have one (AAA-1) or two non-identical modules AAA (AAA-1, AAA-2; nucleotide binding domains). Each of them is composed of two subdomains: the N-terminal that is the core module and the C-terminal domain. The core consists of $200-250$ amino acid residues, which form a characteristic secondary structure conserved in the evolution, the $\alpha-\beta$ - $\alpha$ type $[4,5]$. The core module contains two classical motifs, Walker A $\left(\mathrm{GX}_{4} \mathrm{GKT} / \mathrm{S}\right)$ and Walker B (hy $\left.{ }_{4} \mathrm{DE}\right)$, which are responsible for nucleotide binding and hydrolysis. In addition, the AAA module contains sensor- 1 and sensor- 2 motifs. These motifs are associated with the movement of AAA domains because they link ATP hydrolysis to conformational changes in the substrate binding domains of ClpB protein [6]. The C-terminal domain, located in the core of the AAA module, consists of at least three $\alpha$-helices, and therefore is called the $\alpha$-helix domain. Depending on the number of AAA modules, AAA+ ATPases can be divided into two classes. Class I includes proteins having both AAA-1 and AAA-2 modules (e.g. ClpA, ClpB/Hsp104). The AAA+ ATPases belonging to Class II contain only one AAA module, which corres- 
ponds to AAA-2 (e.g. ClpX, ClpY) [7]. AAA modules tend to oligomerize forming predominantly hexameric ring-shaped structures in which the individual subunits have the same orientation. Subunits of the hexameric ring can communicate with each other and react to conformational changes after nucleotide binding through $\alpha$ helical domain interactions. Hexamers are the biologically active forms of AAA+ proteins. Bacterial ClpB protein contains all characteristic sequence motifs of AAA+ ATPases.

\subsection{Structure, Function and Isoforms of the ClpB Chaperone}

Like other Hsp100 proteins, ClpB forms barrel-shaped hexamers in the presence of ATP [8]. Each ClpB protomer is composed of an N-terminal domain, two ATPbinding modules (NBD-1, NBD-2 or AAA-1, AAA-2 modules), and an inserted coiled-coil middle domain (MD). Both NBD domains have been shown to bind and hydrolyze ATP. ATP binding to the NBD domaines stabilizes oligomeric structure of $\mathrm{ClpB}$ and its interaction with the protein substrates [9,10]. ATP hydrolysis provides energy necessary for remodeling aggregated proteins. The most characteristic elements of the ClpB hexameric ring are a central channel and a long "coiledcoil” segment corresponding to MD located outside the hexamer. The unique MD with a length of about 120 amino acid residues distinguishes the ClpB protein from other Hsp100. It consists of two motifs: Motif 1 that contains helices 1, 2, and Motif 2 that is composed of helices 2, 3 and 4. Each of these motifs resembles a leucine zipper structure that is otherwise characteristic for the known eukaryotic transcription factors. However, unlike the classical leucine zipper motif, Motifs 1 and 2 present in the sructure of ClpB are composed of two anti-parallel $\alpha$-helices with only three consecutive repeats of a leucine occurring every 7 amino acid residues [11]. It has been demonstrated that MD is a mobile element of ClpB. Its position and degree of mobility significantly affect this protein's chaperone activity and in turn the protein disaggregation and reactivation processes [11]. Removal of even a small fragment of MD destabilizes oligomeric states of ClpB and causes loss of its chaperone activity [12]. According to recent reports, MD (in particular Motif 1) mediates the interactions between subunits of ClpB, thus stabilizing the oligomeric structure of the protein; MD also links the ATPase activities of both NBD domains, especially in the presence of a protein substrate [13]. It has been also demonstrated that MD is essential for synergistic interaction with the system Hsp70/DnaK system, and it determines the species specificity of $\mathrm{ClpB} / \mathrm{Hsp} 104$, required for cell suvival at high temperatures [14,15].
Recent finding on biological function of ClpB suggests that the unique chaperone activity of ClpB reversing aggregation is linked to translocation of protein substrates through the narrow central channel within the hexameric ring-shaped structure of ClpB [16].

The ClpB chaperone from Escherichia coli $\left(\mathrm{ClpB}_{\mathrm{Ec}}\right)$ as well as the yeast protein, Hsp104, have been the most intensely studied Hsp100 proteins in recent years because of their unique function in reactivation of protein aggregates formed under stress conditions. The $c l p B_{E c}$ gene contains an internal translation-initiation site and is expressed in vivo as two proteins: a full-length $95-\mathrm{kDa}$ ClpB (ClpB95) and a N-terminal-truncated 80-kDa isoform (ClpB80, ClpB $\Delta N$ ) [17] (Figure 1). The internal initiation site is located near the $\mathrm{N}$-terminus of the first nuclotide-binding domain in ClpB. Thus, the N-terminal domain of ClpB95 is not present in ClpB80. The biological function of the $\mathrm{N}$-terminal truncated isoform in reactivation of protein aggregates has been discovered relatively recently [18]. It was found that ClpB95 and ClpB80 cooperate in reactivation of proteins and form a highly efficient chaperone system. It is assumed that the functional cooperation between $\mathrm{ClpB}$ isoforms arises from interactions between them, because ClpB95 and ClpB80 associate into hetero-oligomers, which form with a higher efficiency than the homo-oligomers of ClpB95. Thus, it is very likely that the ClpB95/80 hetero-association boosts the aggregate-reactivation potential of $\mathrm{ClpB}$ and that $E$. coli produces two variants of $\mathrm{ClpB}$ to optimize its activity. The two isoforms of $\mathrm{ClpB}$ chaperone acting together also enhanced the solubility and enzymatic activity of $\beta$-galactosidase trapped in inclusion bodies [19]. This result supports an important role of synergistic cooperation between the two ClpB isoforms in aggregate reactivation.

The discovery of disaggregation activity of ClpB (Hsp104) chaperone revealed new therapeutic possibilities. For example it gave rise to new strategies in the treatment of neurodegenerative diseases. It was shown that the insoluble aggregates, which appear in neuronal cells and are associated with neurodegenerative diseases, con-

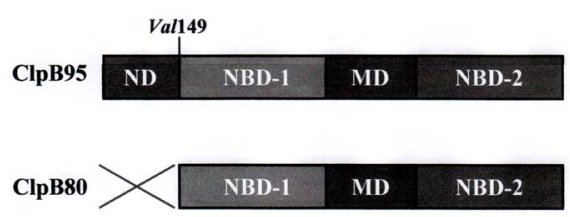

Figure 1. Domain organization of the ClpB95 and ClpB80 monomers. The diagram shows structural domains of the two ClpB isoforms: N-terminal domain (ND), nucleotide-binding domain 1 (NBD-1), middle coiled-coil domain (MD) and nucleotide-binding domain 2 (NBD-2). ND of ClpB95 is not present in ClpB80. 
tain, besides Hsp70 and Hsp40, such proteins as: VCP/ p97, torsin A and the 19S proteasomal complexes. These proteins belong to the AAA+ family of proteins similarly to ClpB. Currently, it is believed that chaperones play a protective role in neurodegenerative diseases. However, there may be another side of the chaperone function (chaperone-linked negative side effect). Namely, ClpB has been shown to be up-regulated in many pathogenic bacteria during infection of the host organism, which helps the pathogen to overcome host defenses.

\section{ROLE OF THE CLPB MOLECULAR CHAPERONE IN PATHOGENIC BACTERIA-OTHER ASPECT OF ITS FUNCTION}

The data accumulated over the last few years regarding several bacterial pathogens suggest that $\mathrm{ClpB}$ chaperone may play an important role in their virulence. However, currently it is not clear what specific role ClpB plays during bacterial infection. ClpB has been shown to be required for invasiveness and/or survival of many pathogens such as Mycoplasma pneumoniae, Porphyromonas gingivalis, Listeria monocytogenes, Mycobacterium tuberculosis, and Francisella tularensis [20-24]. The clpB mutant of Leptospira interrogans has been also shown to be avirulent compared to its parental strains [25]. The loss of ClpB function in L. interrogans has also resulted in bacterial growth defects under oxidative and heat stresses. The authors demonstrated that the Leptospira ClpB is essential for survival under stress conditions and also during an in vivo infection. Pathogenic bacteria are exposed to a significant stress during infection of the host, frequently resulting in protein aggregation. The fact that $\mathrm{ClpB}$ is usually up-regulated in pathogens suggests that ClpB activity as a protein disaggregase may be important for their virulence. In the case of pathogens such as Mycoplasma pneumoniae and Francisella tularensis it has been also shown that ClpB elicits immune responsiveness in experimentally infected laboratory animals $[20,26]$. Similarly, human sera collected from patients suffering from tularemia or infected with $M$. pneumoniae strongly recognized $\mathrm{ClpB}$ chaperone in contrast to uninfected controls. Our studies on the biolgical function of the ClpB from other pathogenic bacterium, Leptospira interrogans, and its potential role in pathogenesis of leptospirosis also suggest that $\mathrm{ClpB}$ of bacterial pathogens possesses an immunogenic potential. We have found that a Leptospira recombinant ClpB protein was recognized by sera collected from infected animals (unpublished data), and thus it is able to activate the host immune system. All of these results may indicate the involvement of $\mathrm{ClpB}$ function in the infection process.

Sequence alignment of $\mathrm{ClpB}_{\mathrm{Ec}}$ and $\mathrm{ClpB}$ from some bacterial pathogens using the Clustal software shows that ClpB proteins of pathogenic bacteria have a similar domain organization to that of $\mathrm{ClpB}_{\mathrm{Ec}}$ with all charasteristic AAA+ motifs (Walker A, Walker B, sensor-1, sensor-2). For example, the total sequence identity between $\mathrm{ClpB}_{\mathrm{Ec}}$ and the Leptospira interrogans $\mathrm{ClpB}$ is $52 \%(27.7 \%$ within ND, 45.3\% within MD, 72\% within NBD-1, and $65.7 \%$ within NBD-2). Similarity with orthologs from the pathogen $L$. borgpetersenii and the saprophyte $L$. biflexa is $95 \%$ and $62 \%$, respectively [25]. It is tempting to speculate that the low sequence similarity between Leptospira interrogans ClpB and ClpB from the saprophytic species, $L$. biflexa, indicates the presence of a yet unknown element in this protein, that can determine ClpB's virulent properties. It would be intriguing to compare in greater detail the biochemical properties of $\mathrm{ClpB}$ proteins from the pathogenic and saprophytic species of Leptospira.

To date, there is only one published report on detailed biochemical studies of ClpB activity isolated from a pathogenic bacterium [27]. These studies were done by Prof. Zolkiewski's group with Ehrlichia chaffeensis $\mathrm{ClpB}$ and show that there are several differences between species in the functioning of ClpB proteins. It was found that the basal ATPase activity of Ehrlichia chaffeensis ClpB was similar to that of the $\mathrm{ClpB}_{\mathrm{Ec}}$. However, while the ATPase of $\mathrm{ClpB}_{\mathrm{Ec}}$ was activated in the presence of casein or poly-lysine, the ATPase of ClpB from Ehrlichia did not respond to the activators. Moreover, it was demonstrated that: 1) Ehrlichia ClpB can reactivate some aggregated substrates even in the absence of the DnaK system, while $\mathrm{ClpB}_{\mathrm{Ec}}$ requires the co-chaperones to disaggregate a substrate; 2) the ClpB from Ehrlichia does not rescue the growth-defect of the $c l p B$-null $E$. coli under heat-shock. Thus Ehrlichia ClpB can not function in the E. coli cells and substitute for $\mathrm{ClpB}_{\mathrm{Ec}}$. A lack of complementation effect may confirm species-specificity of ClpB proteins and suggests that the substrates recognized by ClpB of pathogens during infection of the host differ from the proteins aggregated upon heat shock in E. coli cells. Such result could prove functional differences between the ClpB proteins from pathogenic bacteria and $E$. coli. Futher studies are needed to clarify the role of ClpB chaperone as a virulence factor in bacterial infectious diseases. It is worth to note that $\mathrm{ClpB}$ is not the only chaperone involved in virulence of pathogenic bacteria. For example, synthesis of two other major chaperones, DnaK and GroESL from Staphylococcus aureus, has been shown to be induced during infection of human epithelial cells [28]; also DnaK from Listeria monocytogenes is required for survival under stress conditions and efficient phagocytosis with macrophages [29]. Similarly, the highly conserved ClpB family has been demonstrated to play an important role in the virulence of many pathogenic 
bacteria. The ClpC and ClpXP from Porphyromonas gingivalis have been found to be essential for survival under heat shock and during entry into host epithelial cells [21]. All of these data suggest that molecular chaperones play importat roles in invasion and virulence of bacterial pathogens, as 1) they help the pathogen to overcome stressfull conditions to which it is exposed within the host cells, and 2) they represent major targets of the host's immune system.

Since it is known that ClpB does not exist in mammals, and taking into account its potential role in virulence, it is reasonable to consider it as a potential therapeutic target in antibacterial therapies. Thus, ClpB offers new hope in the fight against pathogenic bacteria. Understanding the function of ClpB in pathogens and dissecting its biochemical properties may improve the development of treatment of several major bacterial diseases, particularly those which do not respond to conventional antibiotics. For treatment of diseases caused by pathogenic bacteria, interfering with the function of the $c l p B$ gene could provide a new direction in antimicrobial therapies as taking $\mathrm{ClpB}$ as a therapeutic target could be more successful than using currently available antibiotics. Therefore, studies on the biological role and biochemical mechanism of ClpB in pathogens are so important and should be continued to demonstrate if $\mathrm{ClpB}$ is in fact important during infection due to its role as molecular chaperone taking part in reactivation of protein aggregates.

\section{ACKNOWLEDGEMENTS}

We thank Katarzyna Potrykus for critical reading of the manuscript and helpful discussion.

\section{REFERENCES}

[1] Zolkiewski, M. (1999) ClpB cooperates with DnaK, DnaJ, and GrpE in suppressing protein aggregation. The Journal of Biological Chemistry, 274, 28083-28086. http://dx.doi.org/10.1074/jbc.274.40.28083

[2] Squires, C.L., Pedersen, S., Ross, B.M. and Squires, C. (1991) ClpB is the Escherichia coli heat shock protein F84.1. Journal of Bacteriology, 173, 4254-4262.

[3] Neuwald, A.F., Aravind, L., Spouge, J.L. and Koonin, E.V. (1999) AAA+: A class of chaperone-like ATPases associated with the assembly, operation, and disassembly of protein complexes. Genome Research, 9, 27-43.

[4] Hanson, P.I. and Whiteheart, S.W. (2005) AAA+ proteins: Have engine, will work. Nature Reviews Molecular Cell Biology, 6, 519-529. http://dx.doi.org/10.1038/nrm1684

[5] Ogura, T. and Wilkinson, A.J. (2001) AAA+ superfamily ATPases: Common structure-diverse function. Genes Cells, 6, 575-597. http://dx.doi.org/10.1046/j.1365-2443.2001.00447.x
[6] Maurizi, M.R. and Li, C.C. (2001) AAA proteins: In search of a common molecular basis. EMBO Reports, 2, 980-985.

[7] Dougan, D.A., Mogk, A., Zeth, K., Turgay, K. and Bukau, B. (2002) AAA+ proteins and substrate recognition, it all depends on their partner in crime. FEBS Letters, 529, 610. http://dx.doi.org/10.1016/S0014-5793(02)03179-4

[8] Akoev, V., Gogol, E.P., Barnett, M.E. and Zolkiewski, M. (2004) Nucleotide-induced switch in oligomerization of the AAA+ ATPase ClpB. Protein Science, 13, 567-574. http://dx.doi.org/10.1110/ps.03422604

[9] Weibezahn, J., Schliker, C., Bukau, B. and Mogk, A. (2003) Characterization of a trap mutant of the AAA+ chaperone ClpB. The Journal of Biological Chemistry, 278, 32608 -32617. http://dx.doi.org/10.1074/jbc.M303653200

[10] Schlieker, C., Weibezahn, J., Patzelt. H., Tessarz, P., Strub, C., Zeth, K., Erbse, A., Schneider-Mergener, J., Chin, J.W., Schultz, P.G., Bukau, B. and Mogk, A. (2004) Substrate recognition by the AAA+ chaperone ClpB. Nat. Struct. Journal of Molecular Biology, 11, 607-615. http://dx.doi.org/10.1038/nsmb787

[11] Lee, S., Sowa, M.E., Watanabe, Y.H., Sigler, P.B., Chiu. W., Yoshida. M. and Tsai, F.T. (2003) The structure of ClpB: a molecular chaperone that rescues proteins from an aggregated state. Cell, 115, 229-240. http://dx.doi.org/10.1016/S0092-8674(03)00807-9

[12] Kędzierska, S., Akoev, V., Barnett, M.E. and Zolkiewski, M. (2003) Structure and function of the middle domain of ClpB from Escherichia coli. Biochemistry, 42, 1424214248. http://dx.doi.org/10.1021/bi035573d

[13] Del Castillo, U., Alfonso, C., Acebrón, S.P., Martos, A., Moro, F., Rivas, G. and Muga, A. (2011) A quantitative analysis of the effect of nucleotides and the $\mathrm{M}$ domain on the association equilibrium of ClpB. Biochemistry, 50, 1991-2003.

[14] Sielaff, B. and Tsai, F.T. (2010) The M-domain controls Hsp104 protein remodeling activity in an Hsp70/ Hsp40dependent manner. Journal of Molecular Biology, 402, 30-37. http://dx.doi.org/10.1016/j.jmb.2010.07.030

[15] DeSantis, M.E. and Shorter, J. (2012) The elusive middle domain of Hsp104 and ClpB: Location and function. Biochimica et Biophysica Acta, 1823, 29-39. http://dx.doi.org/10.1016/j.bbamcr.2011.07.014

[16] Weibezahn, J., Tessarz, P., Schlieker, C., Zahn, R., Maglica. Z., Lee, S., Zentgraf, H., Weber-Ban, E.U., Dougan, D.A., Tsai, F.T., Mogk, A. and Bukau, B. (2004) Thermotolerance requires refolding of aggregated proteins by substrate translocation through the central pore of ClpB. Cell, 119, 653-665.

http://dx.doi.org/10.1016/j.cell.2004.11.027

[17] Squires, C.L., Pedersen, S., Ross, B.M. and Squires, C. (1991) ClpB is the Escherichia coli heat shock protein F84.1. Journal of Bacteriology, 173, 4254-4262.

[18] Nagy, M., Guenther, I., Akoyev, V., Barnett, M.E., Zavodszky, M.I., Kedzierska-Mieszkowska, S. and Zolkiewski, M. (2010) Synergistic cooperation between two ClpB isoforms in aggregate reactivation. Journal of $\mathrm{Mo}$ lecular Biology, 396, 697-707. http://dx.doi.org/10.1016/j.jmb.2009.11.059 
[19] Guenther, I., Zolkiewski, M. and Kedzierska-Mieszkowska, S. (2012) Cooperation between two ClpB isoforms enhances the recovery of the recombinant $\beta$-galactosidase from inclusion bodies. Biochemical and Biophysical Research Communications, 426, 596-600. http://dx.doi.org/10.1016/j.bbrc.2012.08.135

[20] Kannan, T.R., Musatovova, O., Gowda, P. and Baseman, J.B. (2008) Characterization of a unique ClpB protein of Mycoplasma pneumoniae and its impact on growth. Infection and Immunity, 76, 5082-5092. http://dx.doi.org/10.1128/IAI.00698-08

[21] Capestany, C.A., Tribble, G.D., Maeda, K., Demuth, D.R. and Lamont, R.J. (2008) Role of the Clp system in stress tolerance, biofilm formation, and intracellular invasion in Porphyromonas gingivalis. Journal of Bacteriology, 190, 1436-1446. http://dx.doi.org/10.1128/JB.01632-07

[22] Chastanet, A., Derre, I., Nair, S. and Msadek, T. (2004) clpB, a novel number of the Listeria monocytogenes CtsR regulon, is involved in virulence but not in general stress tolerance. Journal of Bacteriology, 186, 1165-1174. http://dx.doi.org/10.1128/JB.186.4.1165-1174.2004

[23] Estorninho, M., Smith, H., Thole, J., Harders-Westerveen, J., Kierzek, A., Butler, R.E., Neyrolles, O. and Stewart, G.R. (2010) ClgR regulation of chaperone and protease systems is essential for Mycobacterium tuberculosis parasitism of the macrophage. Microbiology, 156, 3445-3455. http://dx.doi.org/10.1099/mic.0.042275-0

[24] Meibom, K.L., Dubail, I., Dupuis, M. et al. (2008) The heat-shock protein ClpB of Francisella tularensis is in- volved in stress tolerance and is required for multiplication in target organs of infected mice. Microbiology, 67, 1384-1401.

http://dx.doi.org/10.1111/j.1365-2958.2008.06139.x

[25] Lourdault, K., Cerqueira, G.M., Wunder Jr, E.A. and Picardeau, M. (2011) Inactivation of clpB in the pathogen Leptospira interrogans reduces virulence and resistance to stress conditions. Infection and Immunity, 79, 3711-3717. http://dx.doi.org/10.1128/IAI.05168-11

[26] Havlasova, J., Hemychowa, L., Brechta, M., Hubalek, M., Lenco, J., Larsson, P., et al. (2005) Proteomic analysis of anti-Francisella tularensis LVS antibody response in murine model of tularemia. Proteomics, 5, 2090-2103. http://dx.doi.org/10.1002/pmic.200401123

[27] Zhang, T., Kedzierska-Mieszkowska, S., Liu, H., Cheng, C., Ganta, R.R. and Zolkiewski, M. (2013) Aggregatereactivation activity of the molecular chaperone ClpB from Ehrlichia chaffeensis. PLOS One, 8, E62454. http://dx.doi.org/10.1371/journal.pone.0062454

[28] Qoronfleh, M.W., Bortner, C.A., Schwartzberg, P. and Wilkinson, J. (1998) Enhanced levels of Staphylococcus aureus stress protein GroEL and DnaK homologs early in infection of human epithelial cells. Infection and Immunity, 66, 3024-3027.

[29] Hanawa, T., Fukuda, M., Kawakami, H., Hirano, H., Kamiya, S. and Yamamoto, T. (1999) The Listeria monocytogenes DnaK chaperone is required for stress tolerance and efficient phagocytosis with macrophages. Cell Stress Chaperones, 4, 118-128. 